\title{
Photoionization Modelling of the Giant Broad-Line Region in NGC 3998
}

\author{
Nick Devereux ${ }^{1 \star}$ \\ ${ }^{1}$ Embry-Riddle Aeronautical University, 3700 Willow Creek Road, Prescott, AZ 8301, USA
}

Accepted 2017 September 22. Received 2017 August 20; in original form 2017 May 25

\begin{abstract}
Prior high angular resolution spectroscopic observations of the Low-ionization nuclear emission-line region (Liner) in NGC 3998 obtained with the Space Telescope Imaging Spectrograph (STIS) aboard the Hubble Space Telescope (HST) revealed a rich UV-visible spectrum consisting of broad permitted and broad forbidden emission lines. The photoionization code XSTAR is employed together with reddening-insensitive emission line diagnostics to constrain a dynamical model for the broad-line region (BLR) in NGC 3998. The BLR is modelled as a large $\mathrm{H}^{+}$region $\sim 7 \mathrm{pc}$ in radius consisting of dust-free, low density $\sim 10^{4} \mathrm{~cm}^{-3}$, low metallicity $\sim 0.01 Z / Z \odot$ gas. Modelling the shape of the broad $\mathrm{H} \alpha$ emission line significantly discriminates between two independent measures of the black hole $(\mathrm{BH})$ mass, favouring the estimate of de Francesco et al. (2006). Interpreting the broad $\mathrm{H} \alpha$ emission line in terms of a steady-state spherically symmetric inflow leads to a mass inflow rate of $1.4 \times 10^{-2}$ $\mathrm{M}_{\odot} / \mathrm{yr}$, well within the present uncertainty of calculations that attempt to explain the observed X-ray emission in terms of an advection-dominated accretion flow (ADAF). Collectively, the model provides an explanation for the shape of the $\mathrm{H} \alpha$ emission line, the relative intensities and luminosities for the $\mathrm{H}$ Balmer, [O III], and potentially several of the broad UV emission lines, as well as refining the initial conditions needed for future modelling of the ADAF.
\end{abstract}

Key words: galaxies: Seyfert, galaxies: individual (NGC 3998)

\section{INTRODUCTION}

NGC 3998, the quintessential Liner (Heckman 1980), is remarkable because high angular resolution spectra obtained with $H S T$ reveal not only broad permitted emission lines but also similarly broad forbidden emission lines suggesting a gas density $\sim 10^{4} \mathrm{~cm}^{-3}$. Such a low gas density allows the central UV-X-ray source to photoionize a large spherical volume of $\mathrm{H}$, estimated to be $\sim 7 \mathrm{pc}$ in radius (Devereux 2011, hereafter D11). NGC 3998 is classified Liner 1.9 by Ho et al. (1997a) due to the observation of a broad $\mathrm{H} \alpha$ emission line. However, HST/STIS spectra presented in D11 reveal broad $\mathrm{H} \beta$ and $\mathrm{H} \gamma$ emission lines as well. Thus, a Liner 1.2 designation is more appropriate if the type scheme proposed by Whittle (1992) for Seyferts is extended to include NGC 3998.

Segregating various types of AGN spectroscopically involves ratios of forbidden and permitted emission line fluxes (Kewley et al. 2006; Baldwin et al. 1981). Such diagnostic

* E-mail: devereux@erau.edu diagrams are of limited utility because ratios involving these lines depend on the angular size of the region being measured as discussed recently by Maragkoudakis et al. (2014). Nevertheless, according to three diagnostic diagrams in Kewley et al. (2006), the high angular resolution STIS spectra identify the AGN in NGC 3998 ambiguously as an extremely unusual H II type, a Liner, and on the borderline between Seyfert and Liner. The ambiguity surrounding the true nature of the AGN in NGC 3998 motivates a more detailed examination.

The observation of broad Balmer emission lines has led several to propose that NGC 3998 possesses a bonafide broad line region (BLR) (Heckman 1980; Blackman et al. 1983; Ho et al. 1997b; de Francesco et al. 2006; Balmaverde \& Capetti 2014). Neither the broad H Balmer lines nor the adjacent continuum are time-variable, at least on a timescale of $\sim$ 4 years. NGC 3998 is just one of many non-reverberating AGN with an unusually large BLR size (Devereux 2015; Balmaverde \& Capetti 2014; Zhang et al. 2007; Wang \& Zhang 2003). Non-reverberating AGN violate the correlation between BLR size and AGN luminosity established for 
reverberating AGN (Kaspi et al. 2005), posing a problem for all secondary methods of estimating $\mathrm{BH}$ masses that rely on a tight correlation between BLR-size and AGN luminosity (e.g. Vestergaard \& Peterson 2006; Bentz et al. 2013; Ho \& Kim 2015). The broad Balmer lines seen in non-reverberating AGN look very similar to those seen in reverberating AGN. Consequently, there is no easy way to distinguish them but using the BLR size-luminosity correlation established for reverberating AGN to estimate the $\mathrm{BH}$ mass in a non-reverberating AGN like NGC 3998 produces a wildly incorrect result as noted previously by D11.

Because it is not possible to measure the size of the BLR in NGC 3998 using reverberation mapping, other methods have to be employed. One approach demonstrated recently for the low luminosity Seyfert 1 nucleus in NGC 3516 (Devereux 2016) is based on photoionization modelling of a trifecta of key observations related to the $\mathrm{H}^{+}$gas responsible for producing the broad Balmer emission lines, namely the shape of the broad Balmer emission lines, the Balmer line luminosity, and the $\mathrm{H} \alpha / \mathrm{H} \beta$ ratio. Collectively, these three observables can be used to constrain a unique photoionization model for the $\mathrm{H}^{+}$gas. The principal merit of photoionization modelling is that it can be applied to all AGN regardless of whether they are reverberating or not. With a few exceptions (Pancoast et al. 2014a,b, and references therein), reverberation mapping generally yields only one measure of size, the Balmer reverberation radius (Peterson et al. 2004), but photoionization modelling yields two; a well defined inner and outer radius, along with a number of other interesting results concerning the physical conditions of the $\mathrm{H}^{+}$gas. Given the importance of the BLR size-luminosity correlation, a key objective of this study is to employ the photoionization code XSTAR (Kallman \& Bautista 2001) to improve on the BLR size estimated previously (D11) for NGC 3998. A further objective is to explore whether or not photoionization modelling of the BLR is able to discriminate between the two independent measures of the BH mass in NGC 3998 that differ by almost a factor of four (Walsh et al. 2012; de Francesco et al. 2006). One may expect so because the $\mathrm{H}^{+}$gas traces the velocity field to within $\sim 0.02 \mathrm{pc}$ of the BH in NGC 3998, and the outer radius of the $\mathrm{H}^{+}$region depends linearly on BH mass, everything else being equal.

The broader context of this study is to highlight the distinction between reverberating and non-reverberating AGN. One can use the lists of Maiolino \& Rieke (1995); Ho et al. (1997b); Marziani et al. (2003) and Véron-Cetty \& Véron (2006) to infer that reverberating AGN represent less than half of all AGN with broad Balmer lines in a volume limited sample ( $\leq 40 \mathrm{Mpc}$ ). Thus, focussing on reverberating AGN and ignoring the larger population of nonreverberating AGN will inevitably lead to a biased perspective on BLR properties. In this regard, the non-reverberating AGN in NGC 3998 is spectacular because it appears to represent the very antithesis of the standard BLR paradigm articulated most recently by Schnorr-Müller et al. (2016).

The layout of the paper is as follows. Section 2 introduces a new methodology that utilises reddening independent observables to constrain a unique XSTAR photoionization model for the BLR in NGC 3998, the physical properties of which are discussed further in Section 3. Conclusions follow in Section 4.

\section{PHOTOIONIZATION MODELLING}

The photoionization model described in the following for NGC 3998 features gas of low density $\left(\sim 10^{4} \mathrm{~cm}^{-3}\right)$ and high ionization parameter, very different from other photoionization models in the published literature that attempt to explain the broad emission lines of AGN in terms of high density $\left(\geq 10^{9} \mathrm{~cm}^{-3}\right)$, low ionization parameter gas that is contained in a broad-line "cloud" or an ensemble of such "clouds" (Baldwin et al. 1995; Korista \& Goad 2000, 2004). Those models have several shortcomings, the most persistent of which are the origin and confinement of such clouds. They also have difficulty explaining the high electron temperature $(\sim 20,000 \mathrm{~K})$ of photoionized gas in low ionization AGN (Komossa \& Schulz 1997; Ferguson et al. 1997; Richardson et al. 2014). If such "clouds" exist, in all likelihood they would not be in a vacuum but rather surrounded by a lower density interstellar medium that could be easily photoionized by the central UV-X-ray source. Thus, the geometry envisaged here for the BLR in NGC 3998 is akin to an H II region or a planetary nebula, but with a much higher ionization parameter. In the following, XSTAR is used to model photoionization of the $\mathrm{H}^{+}$region anticipated to be responsible for producing the broad Balmer lines seen in NGC 3998.

XSTAR is a computer code designed to model the emergent spectrum of an $\mathrm{H}^{+}$region that is photoionized by a centrally located UV-X-ray source. XSTAR parameters are listed in Table 1, a full description which can be found in the XSTAR documentation ${ }^{1}$. Values are listed in Table 1 only for fixed quantities and initial conditions. Briefly, the parameters describe the shape and amplitude of the ionizing source, and the physical properties of the medium to be ionized.

The model involves a spherically symmetric distribution of neutral $\mathrm{H}$ gas that is photoionized by a centrally located UV-X-ray source. Spherical geometry is suggested by the observation (D11) that the observed Balmer line luminosity is comparable to that expected given the number of ionizing photons available from the central UV-X-ray source implying a high covering factor $\sim 1$ for the BLR gas. A spherical geometry, as opposed to that of a thin disc, is further supported by the observation of single-peak broad Balmer emission lines (D11). The radial number density for such a spherical distribution of neutral gas $\rho(r)$ is represented by a power law of index $n$, normalized by a number density $\rho_{o}$, at a reference radius $r_{o}$, so that $\rho(r)=\rho_{o}\left(r / r_{o}\right)^{-n}$, where $r$ is the radial distance from the central UV-X-ray source. The inner radius of the spherical model is determined by the initial choice of ionization parameter $\xi$ (Tarter et al. 1969),

$\xi=L /\left(\rho r^{2}\right)$

where $L$ is the ionizing luminosity. The ionization parameter used within XSTAR is different than the parameter $U$ employed, for example, by Ferland \& Netzer (1983)

$U=Q(H) /\left(4 \pi \rho r^{2} c\right)$

where $Q(H)$ is the number of $\mathrm{H}$ ionizing photons s ${ }^{-1}$, and c is the speed of light. Converting between $\xi$ and $U$ depends on the frequency dependence of the ionizing continuum $\mathrm{L}_{\nu}$

1 https://heasarc.gsfc.nasa.gov/xstar/docs/html/xstarmanual.html 


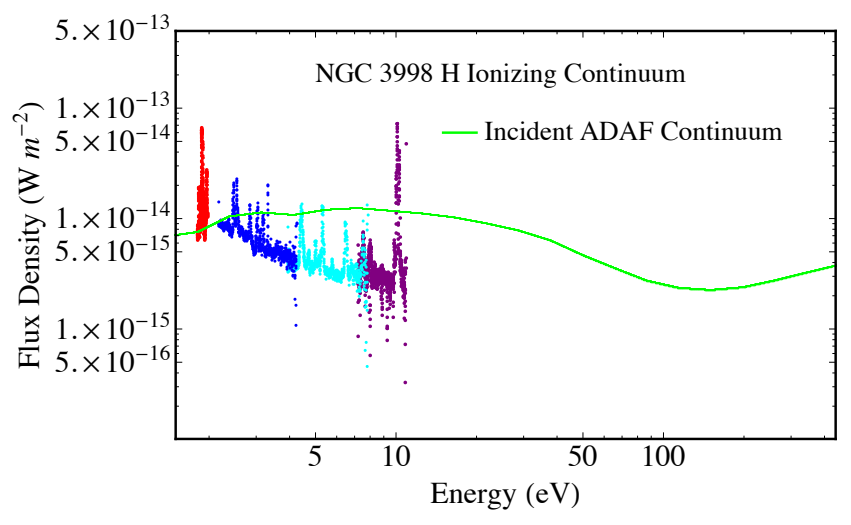

Figure 1. Observed UV-visible continuum of NGC 3998 defined by contemporaneous STIS observations with the G750M, G430L, G230L and G140L gratings, depicted by red, blue, indigo and purple dots, respectively. The solid green line represents the incident ADAF continuum of Nemmen et al. (2014). Units of the ordinate are $\mathrm{W} / \mathrm{m}^{2}$ and the abscissa is $\mathrm{eV}$.

since

$Q(H)=\int_{\nu_{o}}^{\nu_{\max }}\left[L_{\nu} / h \nu\right] d \nu$

On the one hand the continuum emission of NGC 3998 has been interpreted in terms of an ADAF, (Narayan \& Yi 1994) plus jets (Nemmen et al. 2014; Younes et al. 2012; Ptak et al. 2004). On the other hand, Maoz (2007) and Pian et al. (2010) present evidence that the intrinsic visible-UV continuum of Liners may be similar to Seyferts, implicating geometrically thin accretion discs. Yu et al. (2011) demonstrate that the various continuum models actually look very similar to each other and present theoretical arguments in favor of an ADAF origin. Consequently, the ADAF continuum of Nemmen et al. (2014), illustrated in Figure 1, is adopted to represent the intrinsic UV-X-ray continuum of NGC 3998, the luminosity of which corresponds to $9 \times 10^{41} \mathrm{erg} \mathrm{s}^{-1}$ integrated between 1 and 1000 Ryd yielding $7.53 \times 10^{51} \mathrm{H}$ ionizing photons $\mathrm{s}^{-1}$. Thus, $\xi=45 U$.

Neither the gas pressure nor the gas density are held constant for any of the XSTAR models, in contrast to photoionization models of broad-line clouds. Radiation pressure is negligible since the AGN in NGC 3998 radiates at $4 \mathrm{x}$ $10^{-4}$ of the Eddington luminosity limit. An upper limit to the integrated column density of $10^{24} \mathrm{~cm}^{-2}$ was imposed but never achieved for any of the XSTAR models. The XSTAR code runs significantly faster by limiting the suite of elements to those for which emission lines are seen with high confidence in the STIS spectra; H, He, C, N, O, Mg, and S. Also Fe which may be present. Including all 30 elements available within XSTAR changes the model emission line luminosities by $\sim 0.04 \%$ which is inconsequential compared to the measurement uncertainties.

XSTAR produces a comprehensive output including integrated emission line luminosities as well the radial dependence for a number of important physical quantities including the ionization fraction, electron temperature, electron density and ionization parameter. Additionally, each model produced a unique Balmer line emissivity which was used to generate a model $\mathrm{H} \alpha$ emission line profile for comparison
Table 1. XSTAR Input Parameters

\begin{tabular}{ll}
\hline Parameter & Value \\
\hline covering fraction & 1 \\
temperature $\left(10^{4} \mathrm{~K}\right)$ & $\ldots$ \\
constant pressure switch $(1=$ yes, $0=\mathrm{no})$ & 0 \\
pressure $\left(\right.$ dyne $\left.\mathrm{cm}^{-2}\right)$ & $\ldots$ \\
density $\left(\mathrm{cm}^{-3}\right)$ & $\ldots$ \\
spectrum type & file \\
spectrum file & $\ldots$ \\
specrum units? $(0=$ energy, $1=$ photons $)$ & 0 \\
radiation temperature or alpha & $\ldots$ \\
luminosity $\left(10^{38}\right)$ erg $\left./ \mathrm{s}^{-2}\right)$ & $9 \mathrm{e} 3$ \\
column density (atoms cm $\left.{ }^{-2}\right)$ & $1 \mathrm{e} 24$ \\
log (ionization parameter) & $\ldots$ \\
density distribution power law index & $\ldots$ \\
\hline
\end{tabular}

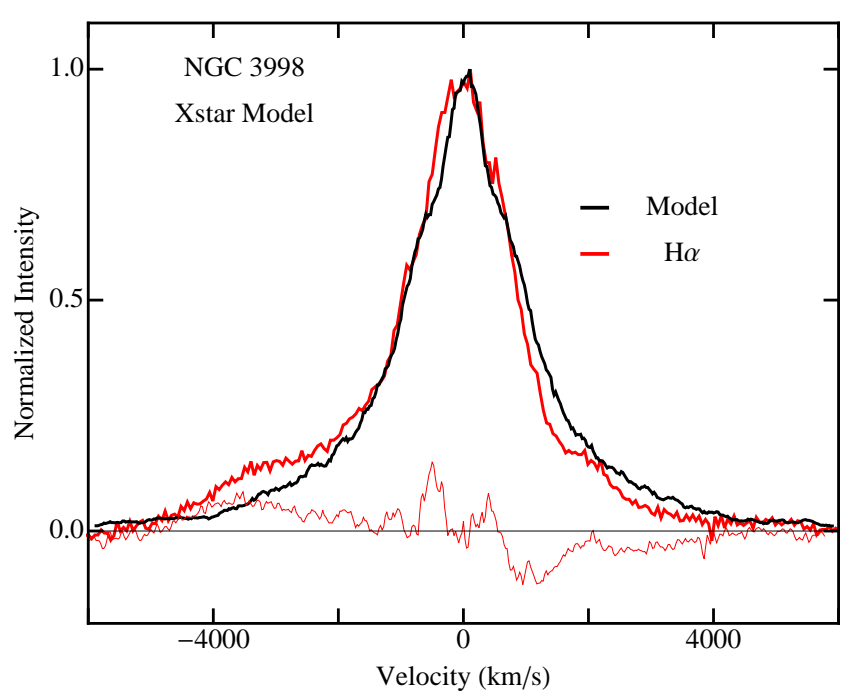

Figure 2. The observed normalized $\mathrm{H} \alpha$ emission line profile is depicted by the red line whereas the XSTAR model is shown in black for $\rho_{o}=3.55 \times 10^{4} \mathrm{~cm}^{-3}, r_{o}=8 \times 10^{-2} \mathrm{pc}$. The residual between the observed and model line is represented by the thinner red line. The abscissa indicates radial velocity relative to rest in $\mathrm{km} / \mathrm{s}$ (see Section 2.1 for details).

with a normalized version of the observed one. Details of the $\mathrm{H} \alpha$ emission line profile modelling are provided in the next section.

\subsection{H $\alpha$ Emission Line Profile}

NGC 3998 was visited twice with STIS and the $\mathrm{H} \alpha$ emission line was observed with the G750M grating on both occasions. Here these two spectra have been combined in order to improve the signal-to-noise ratio. The result reveals a distinctly triangular profile shape illustrated in Figure 2. The $\mathrm{H} \alpha$ emission line is the brightest and best resolved of all the Balmer lines, consequently it is the best line to model.

A model $\mathrm{H} \alpha$ emission line profile is constructed by employing a Monte Carlo simulation of a spherically symmetric 
distribution $^{2}$ of $\sim 10^{4}$ points of light, the radial distribution of which is described by the $\mathrm{H} \alpha$ emission line emissivity. The central mass determines the relationship between velocity and radius for each point of light, and the emissivity determines the number of points at each radius. Thus, knowing both the $\mathrm{H} \alpha$ emission line emissivity and the central mass allows one to construct a model $\mathrm{H} \alpha$ emission line profile given a kinematic description for the BLR gas. Regrettably, the kinematic state of the BLR gas is unknown. However, for the purposes of computing model line profiles, every point of light was assumed to be moving at the escape velocity, which is equivalent in magnitude to the free-fall velocity, according to the familiar equation $v(r)=\sqrt{2 G M(r) / r}$, where $v$ is velocity, $G$ is the gravitational constant, $M(r)$ is the mass interior to $r$, where $r$ is the radial distance of each point from the central supermassive black hole $(\mathrm{BH})$. The radial dependence of the central mass arises because $M(r)$ includes both the $\mathrm{BH}$ mass $M_{\bullet}$, and the surrounding stars $M_{\star}(r)$, which will be important if the BLR gas is spatially extended as appears to be the case for NGC 3998. Such spherically symmetric free-fall models are able to reproduce the distinctly triangular shape observed for the broad $\mathrm{H}$ Balmer lines in NGC 3998, whereas a thin accretion disc would produce two emission peaks unless the disc is contrived to be nearly faceon (D11). Values adopted for $M_{\star}(r)$ are listed in Table 1 and represent the same stellar mass model employed by Walsh et al. (2012, M. Sarzi 2016, private communication) and a distance to NGC 3998 of 14 Mpc (Tonry et al. 2001). Given the likelihood that the BLR gas is spatially extended, no contribution to the model emission line profile was allowed for points of light that lie beyond the angular extent of the STIS slit, corresponding to $52^{\prime \prime} \times 0.1^{\prime \prime}$. The goodness of fit between the model $\mathrm{H} \alpha$ line profile and the observed one is measured using the reduced chi-squared statistic

$\chi_{r e d}^{2}=\sum_{j}\left(O_{j}-M_{j}\right)^{2} /\left(\nu \delta^{2}\right)$

where $O_{j}$ represents the observed normalized line profile intensities, $M_{j}$, the model normalized line profile intensities, $\nu=470$ degrees of freedom and $\delta$ the uncertainty in the observed normalized line profile intensities, taken to be $4 \%$. The summation was performed over the velocity span of the broad $\mathrm{H} \alpha$ line depicted in Figure 2.

\subsection{Photoionization Modelling Results}

The earliest photoionization models of Seyferts and Liners (Ferland \& Netzer 1983; Halpern \& Steiner 1983) implicated low metallicity gas. Subsequently, Binette (1985) noted the connection between the shape of the ionizing continuum and the metal abundance inferred from the $[\mathrm{O}$ III $] \lambda 5008 / \mathrm{H} \beta$ ratio. As explained in more detail in the following, for the ADAF continuum of Nemmen et al. (2014), a low metallicity is required to reproduce the $[\mathrm{O}$ III $] \lambda 5008 / \mathrm{H} \beta$ ratio observed with STIS (D11).

2 As noted in D11 details of the resulting line profile shape are sensitive to the polar and azimuthal angles $\theta$ and $\phi$. Restricting the range of $\theta$ such that $-0.97 \pi / 2 \leq \theta \leq 0.97 \pi / 2$ simulates the cavity expected to be occupied by the radio jets and causes the model profile to more closely mimic the observed profile at zero velocity.
Table 2. Stellar Mass Model of NGC 3998

\begin{tabular}{cccc}
\hline $\begin{array}{c}\text { Radius } \\
\prime\end{array}$ & $\begin{array}{c}\text { Radius } \\
\mathrm{pc} \\
(2)\end{array}$ & $\begin{array}{c}\text { Velocity } \\
\mathrm{km} / \mathrm{s} \\
(3)\end{array}$ & $\begin{array}{c}\mathrm{M}_{\star} \\
\mathrm{M}_{\odot} \\
(4)\end{array}$ \\
\hline $1.0 \times 10^{-1}$ & 7 & 356.4 & $2.0 \times 10^{8}$ \\
$8.7 \times 10^{-2}$ & 6 & 358.4 & $1.7 \times 10^{8}$ \\
$7.3 \times 10^{-2}$ & 5 & 365.1 & $1.5 \times 10^{8}$ \\
$5.8 \times 10^{-2}$ & 4 & 382.4 & $1.3 \times 10^{8}$ \\
$4.4 \times 10^{-2}$ & 3 & 406.3 & $1.3 \times 10^{8}$ \\
$2.9 \times 10^{-2}$ & 2 & 393.3 & $6.9 \times 10^{7}$ \\
$1.4 \times 10^{-2}$ & 1 & 261.9 & $1.5 \times 10^{7}$ \\
\hline
\end{tabular}

A grid of photoionization models spanning a range of metallicity relative to solar $Z / Z_{\odot}$, where $10^{-3} \leq Z / Z_{\odot} \leq$ $10^{-1}$, power law index, $n$, where $0.5 \leq n \leq 1.25$, and gas density $\rho_{o}$, where $4 \leq \log _{10} \rho_{o}\left(\mathrm{~cm}^{-3}\right) \leq 5$, was constructed in order to discover the intersection of model predictions with the observed reddening-insensitive $[\mathrm{O}$ III $] \lambda 5008 / \mathrm{H} \beta$ and $[\mathrm{O}$ III $](\lambda 5008+\lambda 4960) / \lambda 4364$ ratios. $^{3}$ Additionally, each model produced a unique Balmer line emissivity which was used to generate a model $\mathrm{H} \alpha$ emission line profile shape for comparison with a normalized version of the observed one, as described previously in Section 2.1.

Model results for the grid of photoionization models described above are displayed in Figure 3 as a function of gas density $\rho_{o}$ and index $n$ for a metallicity $Z / Z_{\odot}=0.01$. Such a low metallicity is required for the model to reproduce the observed $[\mathrm{O}$ III $] \lambda 5008 / \mathrm{H} \beta$ ratio $=0.65 \pm 0.03(\mathrm{D} 11)$, depicted by the green lines in Figure 3. Incidentally, that ratio qualifies the AGN in NGC 3998 as a Liner 1.2 according to the type scheme proposed for Seyferts by Whittle (1992). Background shaded contours in Figure 3 refer to the $[\mathrm{O} \mathrm{III}](\lambda 5008$ $+\lambda 4960) / \lambda 4364$ ratio, a measure of electron temperature.

An interesting and important result to have emerged from these photoionization models is that the $[\mathrm{O}$ III $] \lambda 5008 / \mathrm{H} \beta$ ratio depends on metallicity $Z / Z_{\odot}$ when the ionization parameter $\xi$ or $\mathrm{U}$ is held constant. The result is illustrated in Figure 4 where [O III $] \lambda 5008 / \mathrm{H} \beta$ $=60 Z / Z_{\odot}$ for a representative row of points in Figure 3 for which $\log _{10} \xi=2.7$. Other model parameters such as the $[\mathrm{O}$ III $](\lambda 5008+\lambda 4960) / \lambda 4364$ ratio which measures the electron temperature, the $\mathrm{H} \alpha / \mathrm{H} \beta$ ratio which is a surrogate for electron density (Devereux 2016), the $\mathrm{H} \beta$ luminosity, $\mathrm{L}_{H \beta}$ a measure of the number of ionizing photons, and $\chi_{\text {red }}^{2}$ a measure of the goodness of fit between the model and observed $\mathrm{H} \alpha$ emission line profile, hardly depend on metallicity, if at all. With hindsight one can see that the $[\mathrm{O}$ III $] \lambda 5008 / \mathrm{H} \beta$ ratio tracks metallicity at fixed $U$ in the photoionization models of Ferland \& Netzer (1983, see their Figure 2). However, the present work shows that the dependence of $[\mathrm{O}$ III $] \lambda 5008 / \mathrm{H} \beta$ on metallicity persists for a range of ionization parameters, $2.2 \leq \log _{10} \xi \leq 3.2$, that are many orders of magnitude higher than typically employed in AGN photoionization models. In the context of the present analysis, the principal utility of the correlation is that the $[\mathrm{O} \mathrm{III}] \lambda 5008 / \mathrm{H} \beta$ ratio very precisely constrains the metallicity of the photoionized gas in NGC 3998 to be

3 All reported wavelengths are vacuum. 


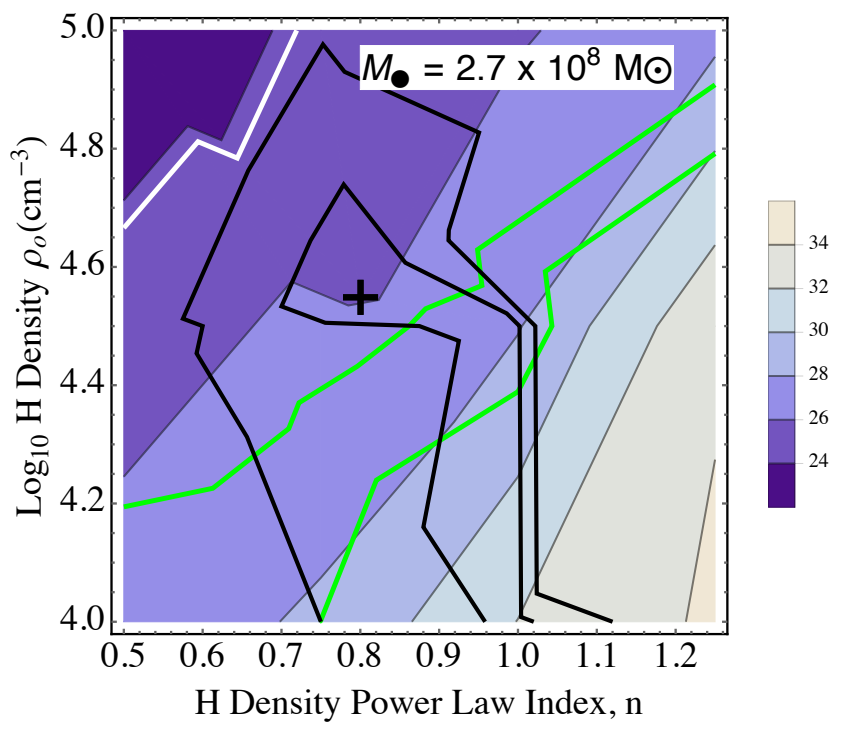

Figure 3. Photoionization model results as a function of gas density $\rho_{o}$ and index $n$ for a metallicity $Z / Z_{\odot}=0.01$. The ordinate identifies the $\mathrm{H}$ density $\rho_{o}$ at a reference radius $r_{o}=2.38 \times 10^{17}$ $\mathrm{cm}$. The abscissa identifies the index $n$ of the power law used to describe the radial distribution of neutral $\mathrm{H}$ gas (see Section 2 for details). The intersection of the model $[\mathrm{O}$ III $] \lambda 5008 / \mathrm{H} \beta$ ratio with the observed value is depicted by the green lines that bound the \pm $1 \sigma$ uncertainties. Lines of constant ionization parameter run parallel to the abscissa. The intersection of the model $[\mathrm{O}$ III] $(\lambda 5008$ $+\lambda 4960) / \lambda 4364$ ratio with the observed value, a lower limit, is depicted by the white line. The legend refers to the background coloured contours depicting the $[\mathrm{O}$ III $](\lambda 5008+\lambda 4960) / \lambda 4364$ ratio, a measure of electron temperature. Black lines identify $\chi_{\text {red }}^{2}$ contours representing the goodness of fit between the model $\mathrm{H} \alpha$ emission line profile and the observed one (see Figure 2 and Section 2.1) for the BH mass $2.7 \times 10^{8} \mathrm{M}_{\odot}$ measured by de Francesco et al. (2006). Two contours are plotted representing $\chi_{\text {red }}^{2}=2$ and $\chi_{\text {red }}^{2}=3$. The black cross represents $\chi_{\text {red }}^{2}=1.2$ and the corresponding model $\mathrm{H} \alpha$ emission line profile is illustrated in Figure 2 .

$0.01 Z / Z_{\odot}$, as illustrated in Figures 3 and 4 . Although not shown, if Figure 3 were reproduced for metallicities bracketing $0.01 Z / Z_{\odot}$, for example $10^{-3} Z / Z_{\odot}$ and $10^{-1} Z / Z_{\odot}$, the figures would look virtually identical to Figure 3 except that the green lines would not be present because the $[\mathrm{O}$ III $] \lambda 5008 / \mathrm{H} \beta$ ratio coincides with the observed one only for $0.01 Z / Z_{\odot}$.

A feature of the models is that a wide range of $\rho_{o}, n$ combinations produce model $\mathrm{H} \alpha$ emission line profiles that mimic the observed one (Figure 2) portrayed by the elongated black contours extending towards low gas densities in Figure 3. Careful examination of these models reveals a degeneracy that includes a wide range of possible sizes for the $\mathrm{H}^{+}$region. However, the predicted ionization structure is such that the outer radius of the $\mathrm{H}^{+}$region coincides with the location of the $\mathrm{O}^{++}$region as illustrated in the upper panel of Figure 5. In this sense the ionization structure is similar to that described by Osterbrock (1989) for a planetary nebula, suggesting a way to break the size degeneracy amongst these various models using the observed [O III] $\lambda 5008$ emission line. A useful observational constraint is that $100 \%$ of the average [O III] $\lambda 5008$ flux measured with

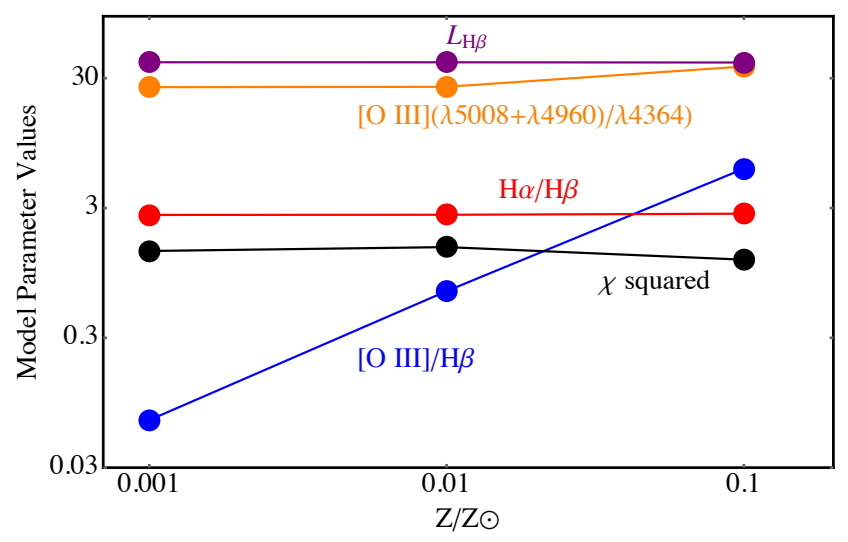

Figure 4. The dependence of various model parameter values on metallicity $Z / Z_{\odot}$ for fixed ionization parameter $\log _{10} \xi=2.7$. The purple line represents $\log _{10}$ of the $\mathrm{H} \beta$ luminosity, $\mathrm{L}_{H \beta}$ in erg $\mathrm{s}^{-1}$, the orange line represents the $[\mathrm{O} \mathrm{III}](\lambda 5008+\lambda 4960) / \lambda 4364$ ratio, a measure of electron temperature, the red line $\mathrm{H} \alpha / \mathrm{H} \beta$ a surrogate of electron density, the black line $\chi_{\text {red }}^{2}$, a measure of the goodness of fit between the model and observed $\mathrm{H} \alpha$ emission line profile, and the blue line represents the $[\mathrm{O}$ III $] \lambda 5008 / \mathrm{H} \beta$ ratio which depends linearly on metallicity.

ground-based telescopes (Heckman 1980; Blackman et al. 1983; Ho et al. 1997a) originates from a region with an angular size comparable to the $0.2^{\prime \prime}$ STIS slit width employed for the G430L observations. Consequently, the size of the $\mathrm{O}^{++}$region, and by association the $\mathrm{H}^{+}$region, must be $\leq$ $0.2^{\prime \prime}$ a constraint that is satisfied, coincidentally, by models that lie above the upper of the two green lines in Figure 3. Thus, a plausible solution, identified by the black cross in Figure 3, that satisfies all three reddening independent observables, corresponds to a gas density $\rho_{o}=3.55 \times 10^{4} \mathrm{~cm}^{-3}$ at $r_{o}=2.38 \times 10^{17} \mathrm{~cm}$ and a power law index $n=0.8$ for the $\mathrm{BH}$ mass, $2.7 \times 10^{8} \mathrm{M}_{\odot}$, measured using gas kinematics by de Francesco et al. (2006). No compelling solutions were found for the BH mass, $8.1 \times 10^{8} \mathrm{M}_{\odot}$, measured using stellar kinematics (Walsh et al. 2012) principally because that $\mathrm{BH}$ mass produced model $\mathrm{H} \alpha$ emission line profiles that consistently did not match the observed one as illustrated by the much larger values of $\chi_{\text {red }}^{2}$ in Figure 6, compared to Figure 3.

The physical dimensions of the BLR in NGC 3998, synonymous now with the $\mathrm{H}^{+}$region, are determined by the $\mathrm{H} \alpha$ emissivity, the radial dependence of which is illustrated in the lower panel of Figure 5. As the UV-X-ray source is approached, the electron temperature increases rapidly at a radius $\sim 0.05 \mathrm{pc}$, where the $\mathrm{H} \alpha$ emissivity concurrently declines because the $\mathrm{H}$ gas is now an X-ray emitting plasma, for which the primary source of opacity is electron scattering. This turnover in the $\mathrm{H} \alpha$ emissivity at small radius provides a natural explanation for the finite width observed for the Balmer lines. The outer radius of the BLR is large, $\sim 7$ pc, corresponding to a sharp decrease in the $\mathrm{H} \alpha$ emissivity that coincides with the outer boundary of the $\mathrm{H}^{+}$region at a $\mathrm{H}$ equivalent column density of $6.5 \times 10^{22}$ atoms $\mathrm{cm}^{-2}$. That the $\mathrm{H} \alpha$ emissivity provides a natural explanation for the overall dimensions of the BLR makes the $\mathrm{H} \alpha$ emission line profile model presented in Section 2.1 all the more com- 

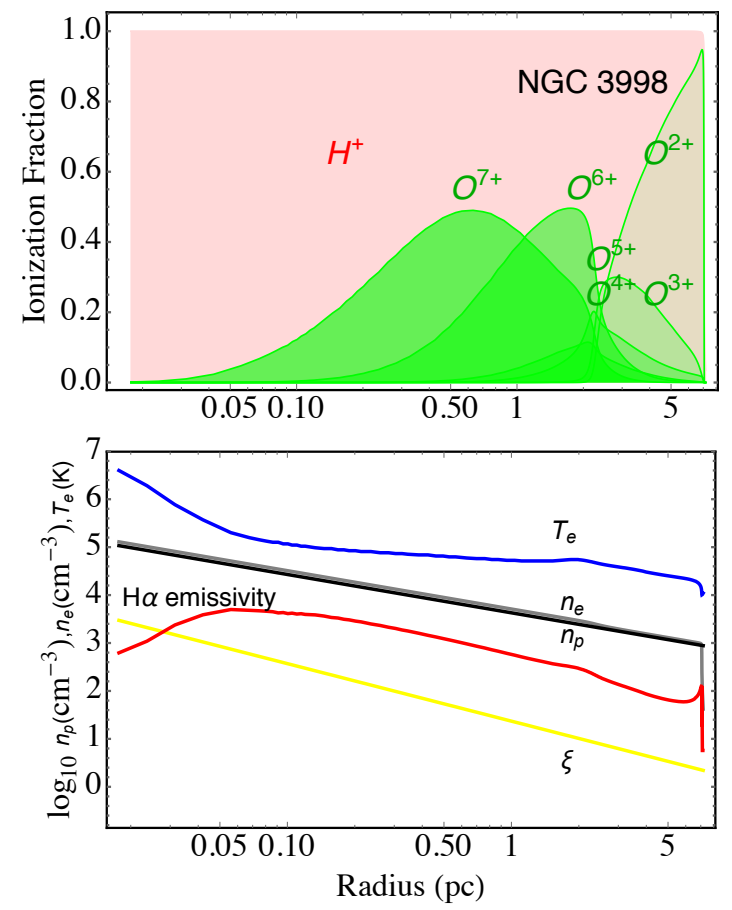

Figure 5. Radial distributions for the model parameters $\rho_{o}=$ $3.55 \times 10^{4} \mathrm{~cm}^{-3}$ at $r_{o}=2.38 \times 10^{17} \mathrm{~cm}, n=0.8, Z / Z_{\odot}=$ 0.01. The upper panel shows the ionization fractions of $\mathrm{H}^{+}$(pink shading) and all possible ionization stages of $\mathrm{O}$ (green shading). The lower panel illustrates the electron temperature, $\mathrm{T}_{e}(\mathrm{~K})$ (blue line), electron density, $\mathrm{n}_{e}\left(\mathrm{~cm}^{-3}\right)$ (grey line), proton density, $\mathrm{n}_{p}$ $\left(\mathrm{cm}^{-3}\right)$ (black line), the $\mathrm{H} \alpha$ emissivity in arbitrary units (red line) and the ionization parameter $\xi$ (yellow line). The ordinate refers to a variety of units whereas the abscissa indicates distance from the $\mathrm{BH}$ in $\mathrm{pc}$.

pelling, especially when compared to other BLR models in the published literature that require the inner and outer radii to be parameterized.

The upper limit of $28,800 \mathrm{~K}$ for the electron temperature constrained using the observed reddening-insensitive [O III] $\lambda \lambda \lambda$ 5008.24,4960.30,4364.44 lines (D11) is also consistent with the temperature predicted for the $\mathrm{O}^{++}$zone (see Figure 5). In summary, the photoionization model presented for NGC 3998 in Figure 5 provides a natural and straightforward physical explanation for the dimensions of the BLR, and hence the shape of the $\mathrm{H} \alpha$ emission line profile, as well as the electron temperature in the $\mathrm{O}^{++}$zone.

\subsection{Dust Extinction to the BLR and the Central UV Continuum Source.}

Galactic extinction to NGC 3998 is negligible, but there is undoubtedly some dust extinction to the Liner nucleus of NGC 3998 caused by the host galaxy (Eracleous et al. 2010). However, dust extinction internal to the BLR gas must be small at visible wavelengths because the Balmer emission line profile shapes are so similar (D11).

The foreground dust extinction $A_{\lambda}$ may be computed

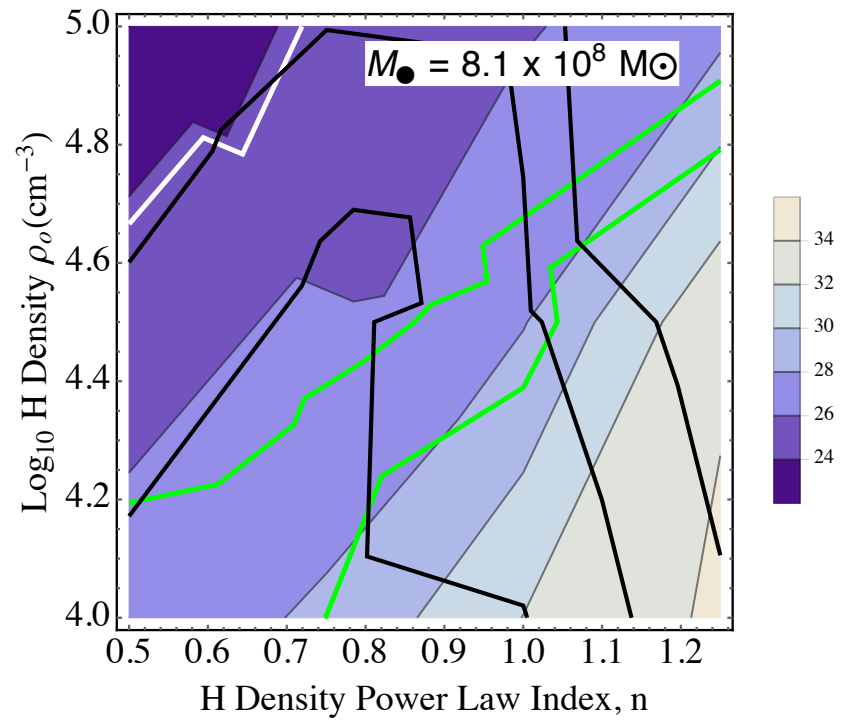

Figure 6. Photoionization model results as a function of gas density $\rho_{o}$ and index $n$ for a metallicity $Z / Z_{\odot}=0.01$. Black lines identify $\chi_{r e d}^{2}$ contours representing the goodness of fit between the model $\mathrm{H} \alpha$ emission line profile and the observed one (see Figure 2 and Section 2.1) for the BH mass, $8.1 \times 10^{8} \mathrm{M}_{\odot}$, measured by Walsh et al. (2012). Three contours are plotted representing $\chi_{\text {red }}^{2}=10,20$ and 30, respectively. Otherwise, the figure and associated legend are identical to Figure 3.

for every observed wavelength $\lambda$ by comparing the observed flux $f_{\lambda}$ with the intrinsic flux $F_{\lambda}$,

$A_{\lambda}=2.5 \log \frac{F_{\lambda}}{f_{\lambda}} \quad \mathrm{mag}$

Subsequently, expressing the total extinction $A_{\lambda}$, as the function of two fixed parameters $\left(A_{\infty}, b\right)$ and one variable, $\lambda$, (Cardelli et al. 1989; Whitford 1958), so that

$A_{\lambda}=A_{\infty}+\frac{b}{\lambda} \quad \mathrm{mag}$

allows one to identify the constant $A_{\infty}$ with the extinction at infinite wavelength, which is expected to be zero. Examples of extinction curves derived using this methodology are presented in Figure 7.

The continuum extinction is derived by adopting the ADAF continuum of Nemmen et al. (2014) as the intrinsic continuum and the contemporaneous STIS observations obtained with the G140L, G230L, G430L and G750M gratings, following removal of the emission lines, as the observed continuum.

The emission line extinction is derived by adopting model predictions for the intrinsic emission line luminosities produced by the $\rho_{o}=3.55 \times 10^{4} \mathrm{~cm}^{-3}$ at $r_{o}=2.38$ $\times 10^{17} \mathrm{~cm}, n=0.8, Z / Z_{\odot}=0.01$ model, which are then compared to the observed $\mathrm{H} \alpha, \mathrm{H} \beta$, and $\mathrm{H} \gamma$ emission line fluxes taken from D11, plus the $\lambda 1640$ He II emission line luminosity which is presented for the first time in Table 3.

Given that the measurement uncertainty is about the same as the point size used in Figure 7, the extinction to the UV-visible continuum is significantly different from that derived using the three brightest Balmer emission lines. An 


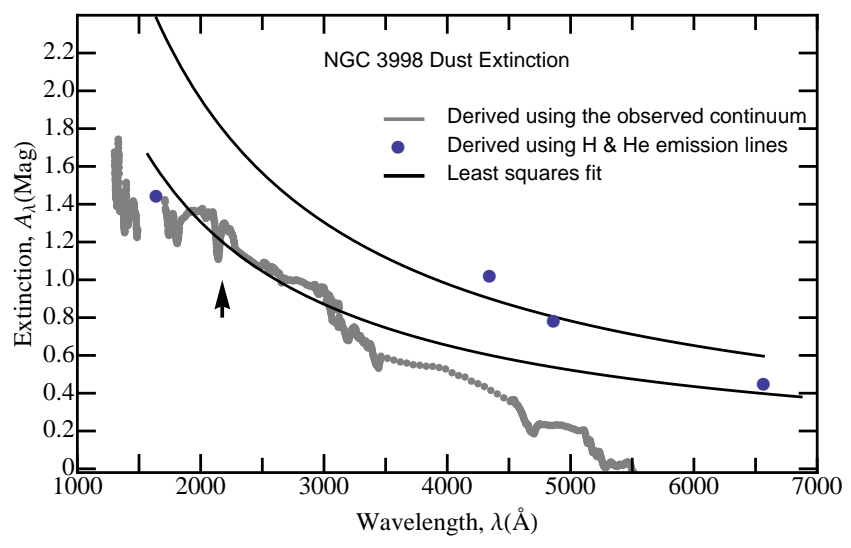

Figure 7. Rest frame extinction to the emission line-free UVvisible continuum in NGC 3998 is defined by comparing contemporaneous STIS observations made with the G140L, G230L, G430L and G750M gratings to the intrinsic ADAF continuum of Nemmen et al. (2014) (light grey line). The grey dots centred at $4000 \AA$ represent an interpolation of the observed continuum over the gap where several emission lines have been removed. Extinction to the $\mathrm{H}^{+}$region is defined by comparing contemporaneous STIS observations of the $\mathrm{H} \alpha, \mathrm{H} \beta, \mathrm{H} \gamma$ and $\lambda 1640$ He II emission lines with the intrinsic emission line fluxes predicted by XSTAR (see Table 3). The ordinate indicates total dust extinction in units of magnitudes. The abscissa indicates wavelength in the rest frame of NGC 3998 expressed in units of $\AA$, and the black arrow identifies the wavelength expected for the $\lambda 2175$ feature.

Table 3. Dust Extinction to Bright H \& He Emission Lines

\begin{tabular}{cccc}
\hline Line & $\begin{array}{c}\text { Observed } \\
10^{39} \mathrm{erg} / \mathrm{s} \\
(2)\end{array}$ & $\begin{array}{c}\text { XSTAR } \\
10^{39} \mathrm{erg} / \mathrm{s} \\
(3)\end{array}$ & $\begin{array}{c}\text { Extinction } \\
\text { mag } \\
(4)\end{array}$ \\
\hline $\mathrm{H} \alpha$ & 10.6 & 16.0 & 0.45 \\
$\mathrm{H} \beta$ & 2.9 & 6.0 & 0.78 \\
$\mathrm{H} \gamma$ & 1.0 & 2.6 & 1.03 \\
$\lambda 1640$ He II & 1.2 & 4.4 & 1.41 \\
\hline
\end{tabular}

unweighted least squares fit to the $\mathrm{H}$ emission lines yields the following extinction law:

$A_{\lambda}=\frac{(3912 \pm 390)}{\lambda(\AA)} \quad \mathrm{mag}$

represented by the upper of the two black curves plotted in Figure 7. According to this extinction law, $E(B-V)=0.17$ \pm 0.01 , slightly higher than the value estimated by Eracleous et al. (2010) based on the $\mathrm{H}$ column required to explain the $\mathrm{X}$-ray absorption.

Interpretation of the UV-visible continuum extinction is more complicated because significant structure exists in the derived extinction. Experimentation revealed that a least squares fit to just the G230L continuum yields the most statistically significant result:

$A_{\lambda}=\frac{(2612 \pm 9)}{\lambda(\AA)} \quad \operatorname{mag}$

represented by the lower of the two black curves plotted in Figure 7 and for which $A_{\mathrm{v}} / E(B-V)=4.3 \pm 0.4$, in the realm of values observed for the Galaxy (Cardelli et al. 1989).

The visible extinction derived using the G430L and G750M continuum observations falls below an extrapolation of the least squares fit to the G230L extinction expressed in Equation 8. This is most likely due to an increasing contribution to the observed visible continuum from starlight at longer wavelengths as noted previously by Nemmen et al. (2014). Another interesting observation, illustrated in Figure 7 , is that the extinction derived using both the $\lambda 1640 \mathrm{He}$ II emission line and the observed G140L continuum flattens in the far UV, $\lambda \leq 2000$. Such greying of the dust opacity has been noted for other AGN as has the absence of the $\lambda 2175$ feature (Gaskell et al. 2004; Gaskell 2017, and references therein).

Finally, XSTAR predicts that aside from $\lambda 1640 \mathrm{HeII}$, the brightest UV emission lines produced inside the $\mathrm{H}^{+}$region are $\mathrm{Ly} \alpha, \lambda 1548 \mathrm{CIV}$, and $\lambda 1906 \mathrm{CIII}$, consistent with the STIS observations presented in D11, although a more detailed analysis is beyond the scope of the present paper, largely because of the absorption associated with Ly $\alpha$ and $\lambda 1548 \mathrm{C}$ IV

\section{DISCUSSION}

$\mathrm{H}$ is the most abundant element in the BLR of NGC 3998 and must be the cornerstone of any viable photoionization model. The $\mathrm{H} \alpha$ emission line, in particular, is sufficiently well resolved in velocity space to pursue sensitive kinematic modelling of the line profile shape using emissivities computed by XSTAR. Photoionization modelling with XSTAR demonstrates that the $\mathrm{H}$ Balmer, [O III] and possibly several of the broad UV emission lines seen in the spectrum of the LINER 1.2 nucleus of NGC 3998 can be explained in terms of a large volume of low density, low metallicity gas that is photoionized by the central UV-X-ray source. The evidence for low metallicity is supported by broad Balmer emission line profile shapes that are observed to be symmetrical and similar to each other (D11) indicating that the $\mathrm{H}^{+}$region must be devoid of $\mu \mathrm{m}$-sized dust grains. These insights result from a novel approach whereby three reddening-insensitive observables, namely the $[\mathrm{O} \mathrm{III}] \lambda 5008 / \mathrm{H} \beta$ and $[\mathrm{O} \mathrm{III]}](\lambda 5008$ $+\lambda 4960) / \lambda 4364$ ratios, plus the $\mathrm{H} \alpha$ emission line profile shape, are used to constrain XSTAR photoionization models which can then be used to infer dust extinction. Usually, observers do the reverse, they attempt to de-redden emission line ratios before using them to constrain photoionization models, but the pitfall with that approach is identifying a suitable reddening indicator at each observed wavelength. The $\mathrm{H} \alpha / \mathrm{H} \beta$ ratio is often used e.g. Heard \& Gaskell (2016); Gaskell (2017, and references therein), but that is a troublesome diagnostic because the ratio is also a proxy for electron density in some AGN (Devereux 2016). Although, having said that, the $\mathrm{H} \alpha / \mathrm{H} \beta$ ratio does appear to be a measure of reddening in NGC 3998 as Figure 7 illustrates.

\subsection{Does NGC 3998 have a BLR?}

NGC 3998 certainly has broad emission lines, but does it have a BLR? Answering this question requires a definition. Laor (2003) proposes that the BLR is the region interior to 

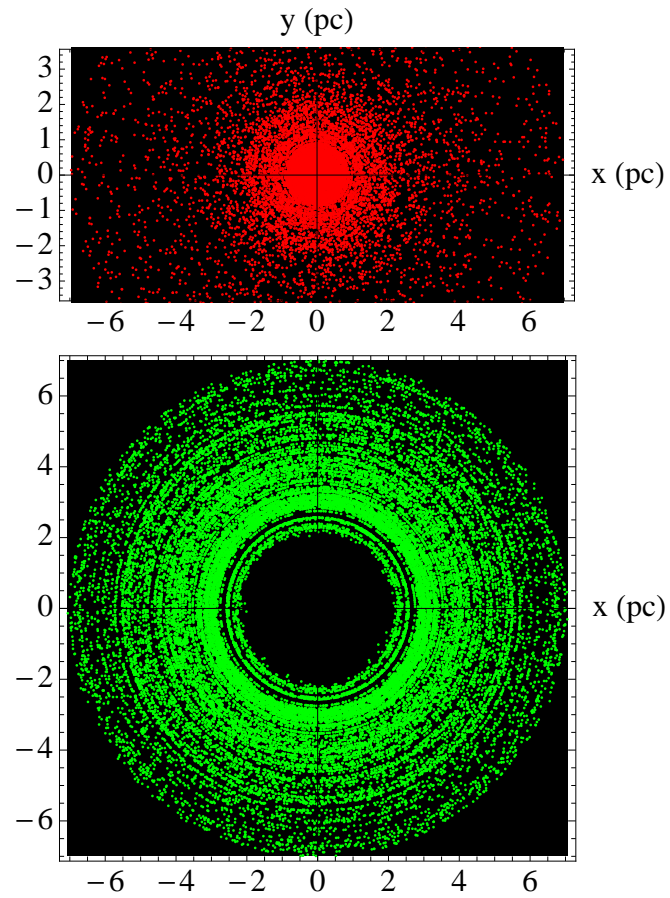

Figure 8. Visualization of the BLR in NGC 3998. Top panel: the ordinate depicts the width, in pc, of the STIS G750M slit used to obtain the $\mathrm{H} \alpha$ observations. Red dots represent the same distribution of points that produced the model $\mathrm{H} \alpha$ emission line illustrated in Figure 2. Lower panel: the ordinate depicts the width, in pc, of the STIS G430L slit used to obtain the [O III] observations. The green dots represent the $[\mathrm{O}$ III $] \lambda 5008$ emissivity. The abscissa illustrates the radial extent of the BLR in both plots in units of pc.

the dust sublimation radius. Although a perfectly reasonable definition, in the case of NGC 3998 it is inadequate for two reasons. First, energy balance considerations (Barvainis 1987) indicate that the luminosity of the central UV-Xray source is insufficient to sublimate dust anywhere inside the $\mathrm{H}^{+}$region responsible for the broad Balmer emission lines. Second, the striking similarity between the $\mathrm{H} \alpha, \mathrm{H} \beta$ and $\mathrm{H} \gamma$ emission lines reported by D11 suggests that the broad Balmer lines arise from a photoionized region that is devoid of dust, presumably because of an intrinsically low metallicity rather than dust sublimation. A more rigorous definition, proposed here, is that the BLR is synonymous with the $\mathrm{H}^{+}$region from where the broad Balmer emission lines emerge. That definition naturally entails an explanation for the physical dimensions of the BLR, at least in NGC 3998 , in terms of the Balmer emissivity as explained previously in Section 2.2.

NGC 3998 also has broad [O III] $\lambda \lambda 5008,4960$ emission lines. Laor (2003) anticipated such an observation if the BLR size-luminosity correlation extends to low luminosity AGN like NGC 3998. Then, as the BLR size decreases, the velocity width of the Balmer lines increases. Because the [O III] emission lines are produced in a region that encircles the BLR they may also broaden by association. The XSTAR results do indeed produce an ionization structure for the AGN in
NGC 3998 that is similar to that envisaged by Laor (2003) as Figure 8 illustrates, but those same results also indicate that the BLR is several orders of magnitude larger than one would expect based on the BLR size-luminosity relationship. The most straightforward interpretation of this result is that the BLR size-luminosity relationship established for reverberating AGN does not apply to non-reverberating AGN like NGC 3998 (Devereux 2015). Part of the size discrepancy results from the observation that the reverberation radius measures only the inner radius of a much larger volume of ionized H gas (Devereux 2016, and references therein). However, even the inner radius of the Balmer emitting region in NGC 3998 ( 0.05 pc, see Section 2.2 and Figure 5$)$ is two orders of magnitude larger than expected. Furthermore, if stars do contribute to the nuclear luminosity in the visible, as the results presented in Section 2.3 indicate, the corrected luminosity will be smaller which will only exacerbate the size discrepancy reported for NGC 3998 by Devereux (2015). Based on the results presented here for NGC 3998, compared to a similar study of the low luminosity Seyfert 1 nucleus in NGC 3516 (Devereux 2016), one can anticipate the density of gas in the BLR to be an important parameter distinguishing non-reverberating AGN from their reverberating counterparts.

\subsection{Comparison of $\mathrm{H}^{+}$and LOC Models}

The XSTAR code is designed to compute the emergent spectrum for a spherical $\mathrm{H}^{+}$region photoionized by a centrally located UV-X-ray source. The resulting ionization structure is such that little or no emission is predicted for low ionization forbidden lines in contradiction to the observed STIS spectrum which clearly shows $[\mathrm{S}$ II], [O II] and [O I] with a full width half maximum velocity that matches the Balmer lines (D11). Regrettably, these forbidden lines are too faint to model, and so the location of the low ionization gas can not be constrained currently using the methodology described in this paper. However, it is quite clear that the low ionization gas must be located outside the $\mathrm{H}^{+}$region. Thus, according to this model there are possibly two separate components, a highly ionized ball of mostly $\mathrm{H}$ surrounded by gas of lower ionization and possibly higher metallicity. Interestingly, the notion of two components was first introduced by Heckman (1980) to explain a curious observation that [O III] emission is seemingly independent of both [O II] and [O I] in Liners and Seyferts.

The model presented here for NGC 3998 predicts the shape of the broad $\mathrm{H} \alpha$ emission line, as well as the relative intensities and luminosities for the $\mathrm{H}$ Balmer, [O III], and potentially several of the broad UV emission lines at the expense of just three free parameters, $\rho_{o}, r_{o}$ and $Z / Z_{\odot}$. This simplicity is to be contrasted with local optimally emitting cloud (LOC) photoionization models of Liners and Seyfert $2 \mathrm{~s}$ which invoke an inhomogeneous interstellar medium characterised by an ensemble of clouds of solar or super-solar metallicity, low ionization parameter, each cloud spanning a wide range of density that is independent of galactocentric radius (Komossa \& Schulz 1997; Ferguson et al. 1997; Richardson et al. 2014). Needless to say, this approach to photoionization modelling is completely different because LOC models aim to explain narrow emission lines observed 
in low angular resolution spectra which display a wide range of ionization.

However, LOC models do have a long-standing problem in that they consistently over-predict the [O III $](\lambda 5008$ $+\lambda 4960) / \lambda 4364$ ratio in low ionization AGN (Komossa \& Schulz 1997; Ferguson et al. 1997; Richardson et al. 2014) whereas the best fitting model for the $\mathrm{H}^{+}$region in the Liner 1.2 nucleus of NGC 3998 predicts an [O III] $(\lambda 5008+$ $\lambda 4960) / \lambda 4364$ ratio that is just $8 \%$ higher than the observed lower limit ${ }^{4}$ (See Section 2.2 and Figure 3). Collectively, the evidence presented supporting a centrally located $\mathrm{H}^{+}$region in NGC 3998 does seem rather compelling and may be worth exploring for other Liners and Seyferts, especially those for which high angular resolution spectra exist. The astrophysics of $\mathrm{H}^{+}$regions is well understood (Osterbrock 1989) and $\mathrm{H}^{+}$regions are a widely observed phenomenon.

Another important difference between LOC and XSTAR models is the geometry employed; slabs versus spheres, or open versus closed geometry. Open geometry predicts emission line flux ratios whereas closed geometry implies a covering factor yielding an important additional constraint, luminosity. Solutions to all photoionization models are highly degenerate. That is to say, for a given metallicity, there are many $\rho_{o}, n$ combinations that give rise to virtually identical emission line ratios and vice versa. However, a temperature and metallicity insensitive luminosity type parameter such as the extinction corrected $\mathrm{H}$ Balmer line luminosity is one important way to break the degeneracy, favouring photoionization models that employ closed geometry.

Finally, the gas pressure gradient in the $\mathrm{H}^{+}$region of the best fitting model is a factor of $\sim 10^{4}$ smaller than required for hydrostatic equilibrium, thus an inflow at the free-fall velocity is inevitable. The term outflow is in widespread use in the professional literature, often with little or no qualification. A true outflow would require gas to exceed the escape velocity. Thus, any outflow velocity that is less than the escape velocity will inevitably become an inflow.

\subsection{Chaotic Cold Accretion}

Assuming spherical symmetry, the BLR of NGC 3998 contains $\sim 36 \times 10^{3} \mathrm{M}_{\odot}$ of ionized $\mathrm{H}^{5}$, estimated by integrating the radial distribution, illustrated in Figure 5, over the radii $2 \times 10^{-2} \leq \mathrm{r}(\mathrm{pc}) \leq 7$. How such a large mass of dustfree low metallicity gas should find its way into the centre of NGC 3998 can be understood in the context of chaotic cold accretion (CCA) described by Gaspari et al. (2013) and Gaspari et al. (2015). Briefly, CCA explains how $10^{4} \mathrm{~K}$ gas can condense out of a $10^{6} \mathrm{~K}$ circumgalactic halo and fall towards the central supermassive BH. In the absence of strong turbulence the inflowing gas is essentially in free-fall. In general, CCA can explain the low duty-cycle observed for AGN and, more specifically for NGC 3998, redirection of the jets powering the larger scale radio lobes (Frank et al. 2016).

Assuming steady-state accretion, a mass inflow rate, $\dot{m}$

${ }^{4}$ caused by blending of the [O III] $\lambda 4364$ emission line with the red wing of the broad $\mathrm{H} \gamma$ emission line. See D11 for details.

5 superseding the lower value estimated by D11 can be estimated using the equation of continuity

$m \dot{(r)}=\epsilon 4 \pi r^{2} v(r) n_{p}(r) m_{p}$

The best fitting model employed a unity filling factor $\epsilon=1$, an inner radius, $r=2 \times 10^{-2} \mathrm{pc}$, where the escape velocity, $v$ is determined by the mass distribution to be $10,950 \mathrm{~km} \mathrm{~s}^{-1}$. The proton mass $m_{p}=1.67 \times 10^{-27} \mathrm{~kg}$. The proton number density $n_{p}=1 \times 10^{5} \mathrm{~cm}^{-3}$ at the inner radius, leading to $\dot{m}$ $=1.4 \times 10^{-2} \mathrm{M}_{\odot} / \mathrm{yr}$, a factor of 4.6 lower than a previous estimate in D11. The revised mass inflow rate is a factor of $\sim 2$ larger than estimated by Nemmen et al. (2014), a factor of 70 larger than estimated by Yu et al. (2011), and a factor of $\sim 4$ larger than that required to explain the observed $\mathrm{X}$ ray emission in terms of metrics provided by Merloni et al. (2003, see D11 for details). The ADAF is located inside the Balmer emitting region, $r \leq 2 \times 10^{-2} \mathrm{pc}$, consistent with the transition radius $R_{t r}$ estimated by Yu et al. (2011) but much smaller than the one adopted by Nemmen et al. (2014). Collectively, the model refines the initial conditions $\dot{m}, R_{t r}$ as well as the physical conditions of the inflowing gas powering the ADAF (Narayan \& Yi 1994). Interestingly, the mass inflow rates calculated above are much higher than estimates for the rate of mass loss produced by the jets (Nemmen et al. 2014; Yu et al. 2011). What becomes of the excess? Hopefully, this question will be answered by examining jet models (e.g. Tchekhovskoy \& Bromberg 2016).

\section{CONCLUSIONS}

A new physical model describes the $\mathrm{H} \alpha$ emission line profile shape, as well as the luminosities and relative intensities of the $\mathrm{H}$ Balmer, [O III], and quite likely several of the broad UV emission lines seen in the STIS spectra of the LINER 1.2 nucleus of NGC 3998, in terms of an $\mathrm{H}^{+}$region consisting of a large volume of low density gas of high ionization parameter that is intrinsically dust-free due to low metallicity. The $\mathrm{H} \alpha$ emission line profile shape is consistent with only the BH mass measured by de Francesco et al. (2006) because the larger BH mass of Walsh et al. (2012) produced model $\mathrm{H} \alpha$ emission line profiles that consistently did not match the observed one. The photoionization calculations indicate that the BLR, synonymous now with the $\mathrm{H}^{+}$region, is large, $\sim 7$ pc in radius, identical to a prior estimate by D11, causing NGC 3998 to not conform to the BLR size-luminosity correlation established for reverberating AGN. The BLR of NGC 3998 contains $\sim 36 \times 10^{3} \mathrm{M}_{\odot}$ of ionized gas. How such a large mass of dust-free low metallicity gas should find its way into the centre of NGC 3998 can be understood in the context of chaotic cold accretion. Collectively, the model refines values of the initial conditions needed for further modelling of the ADAF in NGC 3998, that, in turn, inform jet models.

\section{ACKNOWLEDGEMENTS}

The author thanks Prof. R. Nemmen for the ADAF spectrum illustrated in Figure 1, Dr. Marc Sarzi for providing the stellar mass model presented in Table 2, Dr. Tim Kallman for help with XSTAR, Dr. Steven Willner for patiently reviewing and commenting on many drafts of the manuscript, 
and lastly the referee for thought-provoking questions and comments.

\section{REFERENCES}

Baldwin J. A., Phillips M. M., Terlevich R., 1981, PASP, 93, 5

Baldwin J., Ferland G., Korista K., Verner D., 1995, ApJ, 455, L119

Balmaverde B., Capetti A., 2014, A\&A, 563, A119

Barvainis R., 1987, ApJ, 320, 537

Bentz M. C., et al., 2013, ApJ, 767, 149

Binette L., 1985, A\&A, 143, 334

Blackman C. P., Wilson A. S., Ward M. J., 1983, MNRAS, 202, 1001

Cardelli J. A., Clayton G. C., Mathis J. S., 1989, ApJ, 345, 245

Devereux N., 2011, ApJ, 727, 93

Devereux N., 2015, Journal of Astrophysics and Astronomy, 36, 427

Devereux N., 2016, ApJ, 822, 69

Eracleous M., Hwang J. A., Flohic H. M. L. G., 2010, ApJS, 187, 135

Ferguson J. W., Korista K. T., Baldwin J. A., Ferland G. J., 1997, ApJ, 487, 122

Ferland G. J., Netzer H., 1983, ApJ, 264, 105

Frank B. S., Morganti R., Oosterloo T., Nyland K., Serra P., 2016, A\&A, 592, A94

Gaskell C. M., 2017, MNRAS, 467, 226

Gaskell C. M., Goosmann R. W., Antonucci R. R. J., Whysong D. H., 2004, ApJ, 616, 147

Gaspari M., Ruszkowski M., Oh S. P., 2013, MNRAS, 432, 3401

Gaspari M., Brighenti F., Temi P., 2015, A\&A, 579, A62

Halpern J. P., Steiner J. E., 1983, ApJ, 269, L37

Heard C. Z. P., Gaskell C. M., 2016, MNRAS, 461, 4227

Heckman T. M., 1980, A\&A, 87, 152

Ho L. C., Kim M., 2015, ApJ, 809, 123

Ho L. C., Filippenko A. V., Sargent W. L. W., 1997a, ApJS, 112, 315

Ho L. C., Filippenko A. V., Sargent W. L. W., Peng C. Y., 1997b, ApJS, 112, 391

Kallman T., Bautista M., 2001, ApJS, 133, 221

Kaspi S., Maoz D., Netzer H., Peterson B. M., Vestergaard M., Jannuzi B. T., 2005, ApJ, 629, 61

Kewley L. J., Groves B., Kauffmann G., Heckman T., 2006, MNRAS, 372, 961

Komossa S., Schulz H., 1997, A\&A, 323, 31

Korista K. T., Goad M. R., 2000, ApJ, 536, 284

Korista K. T., Goad M. R., 2004, ApJ, 606, 749

Laor A., 2003, ApJ, 590, 86

Maiolino R., Rieke G. H., 1995, ApJ, 454, 95

Maoz D., 2007, MNRAS, 377, 1696

Maragkoudakis A., Zezas A., Ashby M. L. N., Willner S. P., 2014, MNRAS, 441, 2296

Marziani P., Sulentic J. W., Zamanov R., Calvani M., DultzinHacyan D., Bachev R., Zwitter T., 2003, ApJS, 145, 199

Merloni A., Heinz S., di Matteo T., 2003, MNRAS, 345, 1057

Narayan R., Yi I., 1994, ApJ, 428, L13

Nemmen R. S., Storchi-Bergmann T., Eracleous M., 2014, MNRAS, 438, 2804

Osterbrock D. E., 1989, Astrophysics of gaseous nebulae and active galactic nuclei. University Science Books, Mill Valley, California

Pancoast A., Brewer B. J., Treu T., 2014a, MNRAS, 445, 3055

Pancoast A., Brewer B. J., Treu T., Park D., Barth A. J., Bentz M. C., Woo J.-H., 2014b, MNRAS, 445, 3073

Peterson B. M., et al., 2004, ApJ, 613, 682

Pian E., Romano P., Maoz D., Cucchiara A., Pagani C., Parola V. L., 2010, MNRAS, 401, 677
Ptak A., Terashima Y., Ho L. C., Quataert E., 2004, ApJ, 606, 173

Richardson C. T., Allen J. T., Baldwin J. A., Hewett P. C., Ferland G. J., 2014, MNRAS, 437, 2376

Schnorr-Müller A., et al., 2016, MNRAS, 462, 3570

Tarter C. B., Tucker W. H., Salpeter E. E., 1969, ApJ, 156, 943

Tchekhovskoy A., Bromberg O., 2016, MNRAS, 461, L46

Tonry J. L., Dressler A., Blakeslee J. P., Ajhar E. A., Fletcher A. B., Luppino G. A., Metzger M. R., Moore C. B., 2001, ApJ, 546, 681

Véron-Cetty M.-P., Véron P., 2006, A\&A, 455, 773

Vestergaard M., Peterson B. M., 2006, ApJ, 641, 689

Walsh J. L., van den Bosch R. C. E., Barth A. J., Sarzi M., 2012, ApJ, 753, 79

Wang T.-G., Zhang X.-G., 2003, MNRAS, 340, 793

Whitford A. E., 1958, AJ, 63, 201

Whittle M., 1992, ApJS, 79, 49

Younes G., Porquet D., Sabra B., Reeves J. N., Grosso N., 2012, A\&A, 539, A104

Yu Z., Yuan F., Ho L., 2011, ApJ, 726, 87

Zhang X.-G., Dultzin-Hacyan D., Wang T.-G., 2007, MNRAS, 374,691

de Francesco G., Capetti A., Marconi A., 2006, A\&A, 460, 439

This paper has been typeset from a $\mathrm{T}_{\mathrm{E}} \mathrm{X} / \mathrm{LAT}_{\mathrm{E}} \mathrm{X}$ file prepared by the author. 